\begin{tabular}{|c|l|}
\hline Title & Direct measurement of fluid force on a particle in liquid by telemetry system \\
\hline Author(s) & Harada, Shusaku; Kobay ashi, Y osuke; Saw ano, Takanori; Noguchi, Emi \\
\hline Citation & $\begin{array}{l}\text { International Journal of Multiphase Flow, 37(8), 898-905 } \\
\text { https://doi.org/40.1016/.ijmultiphaseflow.2011.06.010 }\end{array}$ \\
\hline Issue Date & 2011-10 \\
\hline Doc URL & http://hdl.handle.net/2115/47196 \\
\hline Type & article (author version) \\
\hline File Information & IJMF37-8_898-905.pdf \\
\hline
\end{tabular}

Instructions for use 


\title{
DIRECT MEASUREMENT OF FLUID FORCE ON A PARTICLE IN LIQUID BY TELEMETRY SYSTEM
}

\author{
Shusaku Harada $^{\dagger^{* *}}$, Yosuke Kobayashi ${ }^{\dagger}$, Takanori Sawano ${ }^{\dagger}$ and Emi Noguchi ${ }^{\dagger}$ \\ ${ }^{\dagger}$ Division of Sustainable Resources Engineering, Faculty of Engineering, \\ Hokkaido University, N13-W8, Sapporo, Hokkaido, 060-8628, Japan
}

* Corresponding author: Shusaku Harada

Phone/Fax: + (81)-11-7066310

E-mail address: harada@eng.hokudai.ac.jp 


\begin{abstract}
For real-time measurement of the fluid force acting on a particle which moves freely in liquid, we have made a "sensor particle" with a built-in wireless sensor. The sensor particle contains a 3-axis acceleration sensor, 3-axis magnetometer, microcomputer, wireless module and cells. The MEMS acceleration sensor detects the gravitational acceleration in addition to the dynamic acceleration of particle. In order to remove the gravity from acceleration signals, we applied the external magnetic field which is oriented in the same direction as the gravity field. The 3-axis magnetometer detects the direction of external magnetic field and consequently we can remove gravitational acceleration from the sensor signals with arbitrary attitude of particle. A preliminary experiment was performed for checking the accuracy of the measurement system. Firstly we measured the force acting on a settling particle toward a solid wall in liquid. The obtained signals of acceleration (force divided by mass) agrees well with the theoretical prediction of particle motion in liquid for each instant of time. The sensor particle also detects the lubrication force which is exerted on particle for a very short time when it comes close to the wall. Moreover, we applied our system to the gravitational settling of the particle assemblage and detected the fluid force acting on a particle in multi-particle system.
\end{abstract}

Keywords: Liquid-Solid Flow, Particle, Fluid Force, Teremetry System, Sedimentation 


\section{INTRODUCTION}

The behavior of solid particles in liquid is important in various fields such as chemical, mechanical and environmental engineering. It is closely related to many processes as filtration, separation, accumulation, transportation of particulate materials. Although there have been many experimental, theoretical and numerical studies on the dynamics of particles in fluid (Crowe et al, 1998; Brennen, 2005), it has not been understood yet in detail. Two major difficulties prevent us from predicting the behavior of particles. One is particle-fluid interaction and the other is particle-particle interaction. In particular, these interactions are quite complicated in liquid-solid flow in which the density of solid particle is comparable to that of the surrounding fluid.

In most cases, the complicated particle-fluid interaction is caused by unsteady motions of particles relative to the surrounding flow. It is known that unsteady fluid forces, which act on accelerating particles, are significant on the particle motion in a liquid-solid system. The unsteady force acting on an isolated particle can be obtained theoretically in creeping flow (zero Reynolds number) and in inviscid flow (infinite Reynolds number). However, most practical liquid-solid flow is finite Reynolds number flow and particles move with changing the velocity under the influence of surrounding particles. The fluid force exerted on such particles is still unknown except for some theoretical and experimental findings (Brennen, 2005).

Particle-particle interaction is also more complicated in liquid-solid flow compared to that in gas-solid flow. Since the interstitial fluid of particles greatly influences the momentum transfer between them, the particle-particle interaction in liquid is quite different from that in gas (e.g. Barnocky and Davis, 1988; Joseph et al., 2001). The interaction between particles with interstitial fluid effect is called hydrodynamic interaction and it appears as the variance of fluid force acting on each particle. Therefore the basic knowledge of fluid forces on freely-moving particles has been required in order to understand the dynamics of the liquid-solid system.

Measurement systems for detecting particle motion in flow field have been developed in related fields of PIV system (Adrian, 2005). On the other hand, tomographic techniques have come into 
practical use of non-invasive monitoring of particle motion in fluid flow (Chaouki et al., 1997; Kawaguchi, 2010). These systems can detect particle motions in fluid precisely. However, they can obtain only instantaneous particle positions which change every second. Therefore they can not measure the fluid force acting on particles directly and can do nothing but calculate the force via the second derivative of instantaneous position.

In recent years, measuring devices have been downsized by the development of MEMS technology and they have been applied to flow measurements (Ho and Tai, 1998). The downsizing of devices enables innovative approaches to measurement. For instance, a Lagrangian-type sensor, which travels with the flow and transmits signals to outside, has been made for measurement of local temperature in thermal convection flow (Gasteuil et al., 2007; Shew et al., 2007). A similar Lagrangian-type sensor has been developed for measurement of fluid force, however, there is nothing but a prototype system for a fixed particle (Zhang et al., 2009).

In this study, we have developed a "sensor particle" with a built-in MEMS sensor which can measure the fluid force acting on a particle moving freely in liquid. Our system can get real-time and non-invasive measurement of the force acting on particle. This paper describes the design, the measuring principle and the accuracy of the sensor particle we made, and its practical application to liquid-solid system.

\section{EXPERIMENTAL SETUP}

\section{Design of Sensor Particle}

Figure 1 shows the appearance of the sensor particle with a built-in wireless sensor system. The sensor system is composed of a 3-axis acceleration sensor (Hitachi Metals, H34C), a 3-axis magnetometer (Aichi-MI, AMI302), a microcomputer (Microchip, PIC16F877A), a wireless transmitter module (ITEC, iTX315S) and cells (Sony, SR1130). Table 1 indicates operating conditions and characteristics of sensors and wireless modules. The acceleration sensor, the 
magnetometer and the microcomputer are deposited on both sides of a circular substrate $16 \mathrm{~mm}$ in diameter. The wireless module is deposited on the square substrate measuring $11 \mathrm{~mm}$ by $15 \mathrm{~mm}$. These substrates are connected by electronic wires and are put into a spherical shell made from polystyrene. The outer diameter of the shell is $d=30 \mathrm{~mm}$. The shell is openable and divides into two pieces. For waterproof, the joint is sealed by a ring rubber packing from the inside. The mass bias of sensor particle is adjusted by adhesion of clayey silicone paste on the inner surface of the shell. The total mass of sensor particle (including shell, substrates, cells, clayey paste) $m_{p}$ is adjustable by the amount of clayey paste and it ranges from 14.0 to 15.0 gram. The eventual density of sensor particle $\rho_{p}$ is 1015 to $1090 \mathrm{~kg} / \mathrm{m}^{3}$.

Figure 2 shows the circuit diagram of our sensor system. The analog signals obtained from the acceleration sensor and the magnetometer are converted to 10bit digital signals and they are serialized by Universal Synchronous Asynchronous Receiver Transmitter (USART) in microcomputer. The serial signal is sent to transmitter (TX module) and is transmitted wirelessly to receiver (RX module). The received signal by RX module is send to PC through RS-232C cable. In this way, the set of six signals (3-axis acceleration and 3-axis magnetic vectors) is obtained. The sampling speed of the data is determined by the speed of wireless transmission. In our system, the set of signals can be obtained every 31.25 milliseconds and the corresponding sampling frequency is $32 \mathrm{~Hz}$.

\section{Wireless Transmission of Signals}

It is known that the absorption of electromagnetic wave in liquid is much larger than that in air and it greatly depends on the frequency. For example, the absorption coefficient of the electromagnetic wave $\alpha\left[\mathrm{cm}^{-1}\right]$ for frequency $f=2 \mathrm{GHz}$ is approximately unity in pure water, i.e., the wave decays within the order of a few centimeters (Jackson, 1999). On the other hand, the absorption coefficient for $f=300 \mathrm{MHz}$ is $\alpha \sim 10^{-2} \mathrm{~cm}^{-1}$. It means that the travel distance of the wave is the order of a few 
meters in water. Therefore we use a wireless module with radio-frequency of $315 \mathrm{MHz}$, which can send electromagnetic signals from centimeter- to meter-sized vessel to the outside in principle.

The preliminary experiment was performed for checking whether signals are transmitted successfully in water by our system. Figure 3 shows the intensity of received signals from the sensor particle which is fixed in the center of a cubic vessel. The vessel is $0.5 \mathrm{~m}$ per side and it is filled with or without water. We changed the distance between the experimental vessel and RX module (receiver). The signal intensity is obtained from the voltage at the output terminal of $\mathrm{RX}$ module, which is proportional of the intensity of received signals, and it is normalized by the signal when RX module is put just beside the vessel without water. Although the received signals from the vessel with water decay larger than that without water, the signals are successfully transmitted from water vessel which is several dozen centimeters on a side to the outside by our wireless system.

All the measured results shown below are raw data and they do not pass through external noize filters. Concerning the data given here, there is no dropped data. We check the noise of the sensor ststem under quiescent condition and its magnitude reduced to the force is approximately $\pm 0.002 m_{p} g$ ( $m_{p}$ : particle mass, $g$ : gravitational acceleration).

\section{Detection of Dynamic Acceleration}

The acceleration sensor used in this study is 3-axis piezo-resistive type. It detects the gravitational acceleration in addition to the dynamic acceleration as most of the MEMS acceleration sensors. For the measurement of the force acting on a particle, only the dynamic acceleration (force divided by mass) has to be detected. However, it is difficult to remove the gravity and to measure only the dynamic acceleration with arbitrary attitude of particle. In order to overcome this difficulty, we have applied the external magnetic field which is oriented in the same direction as the gravity field.

Figure 4 illustrates the conceptual diagram of our measurement system. The 3 -axis magnetometer can detect the direction of magnetic field even in any attitude of sensor particle. The magnetometer outputs voltages proportional to the components of magnetic vector $\widetilde{\boldsymbol{b}}=\left(\widetilde{b}_{x}, \widetilde{b}_{y}, \widetilde{b}_{z}\right)$ in the 
coordinates $(x, y, z)$ which is fixed on sensor particle. In consequence, the vector of gravitational acceleration $\boldsymbol{g}$ can be calculated as follows;

$$
\boldsymbol{g}=g \frac{\widetilde{\boldsymbol{b}}}{|\widetilde{\boldsymbol{b}}|},
$$

where $g$ is the constant of gravitational acceleration $\left(g=9.8067 \mathrm{~m} / \mathrm{s}^{2}\right)$.

On the other hand, The acceleration sensor outputs the voltages proportional to the vector sum of the dynamic acceleration and the gravitational acceleration in sensor coordinates $\widetilde{\boldsymbol{a}}=\left(\widetilde{a}_{x}, \widetilde{a}_{y}, \widetilde{a}_{z}\right)$. By using Eq.(1), the dynamic acceleration vector $\boldsymbol{a}$ is calculated by subtracting the gravity from the output of acceleration sensor in the following way;

$$
\boldsymbol{a}=\widetilde{\boldsymbol{a}}-g \frac{\widetilde{\boldsymbol{b}}}{|\widetilde{\boldsymbol{b}}|},
$$

The acceleration vector given by Eq.(2) can be obtained as the components in sensor coordinates, i.e., $\boldsymbol{a}=\left(a_{x}, a_{y}, a_{z}\right)$. By calculating the dot product of $\boldsymbol{a}$ and a unit vector of gravitational direction $\tilde{\boldsymbol{b}} /|\widetilde{\boldsymbol{b}}|$, the vertical component $a_{V}$ and horizontal component $a_{H}$ are obtained respectively;

$$
\begin{aligned}
& a_{V}=\widetilde{\boldsymbol{a}} \cdot \frac{\tilde{\boldsymbol{b}}}{|\widetilde{\boldsymbol{b}}|}-g, \\
& a_{H}=\sqrt{|\widetilde{\boldsymbol{a}}|^{2}-\left(\widetilde{\boldsymbol{a}} \cdot \frac{\tilde{\boldsymbol{b}}}{|\widetilde{\boldsymbol{b}}|}\right)^{2}} .
\end{aligned}
$$

\section{External Magnet Field and Error Estimation}

The external magnetic field was made by two planar permanent magnets. The size of each magnetic plate is $425 \mathrm{~mm}$ in length, $425 \mathrm{~mm}$ in width and $20 \mathrm{~mm}$ in height. The separation between two plates is set to be $2200 \mathrm{~mm}$. The experimental vessel (the details are given below) was fixed on the central part between these magnets. We checked the linearity of the magnetic field by preliminary experiment. Figure 5 indicates the magnetic vector between two magnetic plates measured by the 
magnetometer. It is found that the measured magnetic field is not exactly vertical and it has maximum error near the upper and lower magnets. The angle of measured magnetic vector against vertical line $\Delta \theta$ is $10.27 \mathrm{deg}$. at a maximum. However, $\Delta \theta$ is $3-8 \mathrm{deg}$. near the central region at which the experimental vessel is fixed.

If the applied magnetic field is inclined against the gravity field, it causes measurement errors of both vertical and horizontal components of acceleration. Here we check the sensitivity of the measured acceleration by inclined magnetic field. If the magnetic vector is perfectly along with the gravitational vector, the vertical and horizontal components of acceleration are calculated from Eq.(2) as follows.

$$
\begin{aligned}
& a_{V}=|\widetilde{\boldsymbol{a}}| \cos \theta-g, \\
& a_{H}=|\widetilde{\boldsymbol{a}}| \sin \theta,
\end{aligned}
$$

where $\theta$ is the angle of measured acceleration vector $\widetilde{\boldsymbol{a}}$ (dynamic acceleration with gravity) against the vertical line (see inset of Fig.6). If the magnetic vector $\widetilde{b}$ is inclined against the vertical line with the angle $\Delta \theta$, the obtained acceleration components (with error) $a_{V}^{\prime}$ and $a_{H}^{\prime}$ satisfy the following inequalities.

$$
\begin{aligned}
& |\widetilde{\boldsymbol{a}}| \cos (\theta+\Delta \theta)-g \leq a_{V}^{\prime} \leq|\widetilde{\boldsymbol{a}}| \cos (\theta-\Delta \theta)-g, \\
& |\widetilde{\boldsymbol{a}}| \sin (\theta-\Delta \theta) \leq a_{H}^{\prime} \leq|\widetilde{\boldsymbol{a}}| \sin (\theta+\Delta \theta) .
\end{aligned}
$$

Rewriting Eqs.(7) and (8) by using addition theorem of trigonometric functions and Eqs.(5) and (6), we can obtain the following inequalities.

$$
\begin{aligned}
& a_{V}(\cos \Delta \theta-\tan \varphi \sin \Delta \theta)-g(1-\cos \Delta \theta) \leq a_{V}^{\prime} \leq a_{V}(\cos \Delta \theta+\tan \varphi \sin \Delta \theta)-g(1-\cos \Delta \theta), \\
& a_{H}\left(\cos \Delta \theta-\frac{\sin \Delta \theta}{\tan \varphi}\right)-g \sin \Delta \theta \leq a_{H}^{\prime} \leq a_{H}\left(\cos \Delta \theta+\frac{\sin \Delta \theta}{\tan \varphi}\right)-g \sin \Delta \theta,
\end{aligned}
$$

where $\varphi$ is the angle between the dynamic acceleration vector and vertical line and $\tan \varphi=a_{H} / a_{V}$ (see inset of Fig.6). 
As can be seen in Eqs.(9) and (10), the error due to inclined magnetic field affects the acceleration output in two ways, i.e., the error depending on the magnitude of acceleration components (the first term) and the error which is independent on the acceleration (the second term). We can rewrite Eqs.(9) and (10) in the following way.

$$
\begin{aligned}
& C_{V-}(\varphi, \Delta \theta) a_{V}-D_{V}(\Delta \theta) \leq a_{V}^{\prime} \leq C_{V+}(\varphi, \Delta \theta) a_{V}-D_{V}(\Delta \theta), \\
& C_{H-}(\varphi, \Delta \theta) a_{H}-D_{H}(\Delta \theta) \leq a_{H}^{\prime} \leq C_{H+}(\varphi, \Delta \theta) a_{H}-D_{H}(\Delta \theta),
\end{aligned}
$$

where $\quad C_{V \pm}=\cos \Delta \theta \pm \tan \varphi \sin \Delta \theta, \quad C_{H \pm}=\cos \Delta \theta \pm \sin \Delta \theta / \tan \varphi, \quad D_{V}=g(1-\cos \Delta \theta) \quad$ and $D_{H}=g \sin \Delta \theta$. As indicated in Fig.5, the maximum angle of magnetic vector $\Delta \theta$ is around 8 deg. If we set $\Delta \theta=8 \mathrm{deg}$, the above coefficients can be calculated as follows.

$$
C_{V \pm}=0.99 \pm 0.139 \tan \varphi, \quad C_{H \pm}=0.99 \pm \frac{0.139}{\tan \varphi}, \quad D_{V}=0.01 \mathrm{~g}, \quad D_{H}=0.139 \mathrm{~g} .
$$

Given that the minimum detective sensitivity of our sensor system is $\pm 0.005 \mathrm{~g}$, the error due to the constant $D_{V}$ is relatively minor while $D_{H}$ cannot be neglected.

Figure 6 shows the relation between the coefficients $C_{V}, C_{H}$ and the angle $\varphi$ for $\Delta \theta=8$ deg.. As can be seen in Fig.6, $C_{V}$ is not so large for small angle $\varphi$, while the $C_{H}$ is very large. THis suggests that, when the sensor particle moves almost vertically (small $\varphi$ ), the magnetic error has little influence on the measured vertical acceleration $a_{V}$. For example, if the particle moves at $\varphi=15 \mathrm{deg}$. against the vertical axis, the accuracy of vertical acceleration is estimated within $95.3 \%( \pm 0.005 g)$ from Eq.(13), although the accuracy of horizontal acceleration is very poor.

This analysis suggests that, as it stands, our system can be applied to the measurement of vertical fluid force on particulate system where their vertical motions are dominant, i.e., gravitational settling, one-dimensionally fluidized bed and so on. For demonstration of our prototype system, we show the measured results of fluid force in such a vertically moving system in the next section. 


\section{RESULTS AND DISCUSSION}

\section{Fluid Force on a Settling Particle in Liquid}

In order to check the accuracy of our measurement system, we measured the fluid force acting on a settling particle toward a solid wall in liquid. The test vessel is $600 \mathrm{~mm}$ long, $300 \mathrm{~mm}$ wide and 360 $\mathrm{mm}$ high. A solenoid is mounted in the upper part of the vessel as a releasing device for the sensor particle. A tiny iron chip is put on the outer shell of sensor particle. The resulting mass of sensor particle $m_{p}$ is 14.6 gram and the density $\rho_{p}=1060 \mathrm{~kg} / \mathrm{m}^{3}$. The distance between the solenoid and the bottom wall is $208 \mathrm{~mm}$.

The experimental procedure is as follows. At first, silicone oil (density $\rho_{f}=969 \mathrm{~kg} / \mathrm{m}^{3}$, viscosity $\mu_{f}=57.5 \mathrm{mPa} \cdot \mathrm{s}$ at $18.5^{\circ} \mathrm{C}$ ) is filled into the test vessel and the sensor particle is attached on the solenoid. After the particle and fluid are at rest, the solenoid is turned off and the sensor particle starts to settle by gravity. The settling behavior is recorded by digital video camera and the transmitted wireless signal from sensor particle is received by the RX module which is mounted on the outside of the vessel. The obtained acceleration and magnetic signals are immediately sent to the PC. After removing the gravitational acceleration by the way described in Eqs.(1) to (3), the vertical acceleration $a_{V}$ and the vertical force acting on the particle $F=m_{p} a_{V}$ is calculated in real-time.

Models for describing the accelerated motion of particle in liquid have been proposed (Clift et al. 1978). In general, the fluid force acting on a freely-moving particle in flow is quite different from the one on a fixed particle. Nevertheless, most of fluid force models have been derived on the basis of the force on a fixed particle in uniform flow or the force on an accelerating particle in stationary flow. For example, Odar and Hamilton (1964) have proposed the fluid force for accelerated motion of a particle at moderate Reynolds number as follows.

$$
F=\frac{\pi d^{2}}{8} C_{D} \rho_{f} U^{2}+\Delta_{A} \frac{\pi d^{3}}{12} \rho_{f} \frac{d U}{d t}+\Delta_{H} \frac{3 d^{2}}{2} \sqrt{\pi \rho_{f} \mu} \int_{-\infty}^{t} \frac{d U / d t^{\prime}}{\sqrt{t-t^{\prime}}} d t^{\prime}+\frac{\pi d^{3}}{6}\left(\rho_{p}-\rho_{f}\right) g
$$


where $U$ is the particle velocity. The first term of RHS in Eq.(14) indicates the steady drag, the second term is the added mass force, the third is the history force and the fourth is the gravity and buoyant forces. $C_{D}$ is the drag coefficient and the coefficient $\Delta_{A}$ and $\Delta_{H}$ are the empirical coefficients given by

$$
\begin{aligned}
& \Delta_{A}=2.1-\frac{0.132 M_{A}^{2}}{1+0.12 M_{A}^{2},} \\
& \Delta_{H}=0.48+\frac{0.52 M_{A}^{3}}{\left(1+M_{A}\right)^{3}},
\end{aligned}
$$

where $M_{A}$ is the acceleration modulus and $M_{A}=(d U / d t) d / U^{2}$ (Clift et al. 1978). Eqs.(14) to (16) were derived on the assumption of the harmonic motion of a particle and they were made by the modification of the fluid force in creeping flow (zero Reynolds number). Nonetheless, their model can describe well the settling motion of a particle at finite Reynolds number (Odar, 1966).

For comparing the fluid force measured by sensor particle, we integrated numerically the equation of motion with the force given by Eq.(14) and calculated the components of fluid force for each instant of time. The drag coefficient $C_{D}$ was calculated from the empirical relation by Morsi and Alexander (1972). In addition, we considered the effect of the bottom wall by assuming $C_{D}$ to $(24 / R e) d / 2 l_{G}\left(l_{G}\right.$ : the distance between particle and wall) and setting $\Delta_{A}=\Delta_{H}=1$ only in near-wall region. These assumptions means that the fluid force on particle near the wall is replaced with the lubrication force $3 / 2 \pi \mu d^{2} U / l_{G}$. The restitution of particle to the bottom wall was calculated by the collision model taking the lubrication effect into consideration (Barnocky and Davis, 1988; Joseph et al., 2001).

Figure 7 shows the pictures of the settling behavior of sensor particle at intervals of 0.2 seconds. The sensor particle starts to accelerate by gravity after the releasing device is turned off, and then it maintains an almost constant velocity. The particle Reynolds number in this instant is approximately one hundred. Finally the particle collides with the bottom wall at $t=1.2 \mathrm{~s}$ and it slightly rebounds.

Figure 8 (a) indicates the measured results of the force by sensor particle and (b) is the 
theoretical fluid force (except for gravity and buoyant forces) predicted by Eq.(14). The experiment was performed four times under the same conditions. Fig.8 (a) shows that the measured fluid force initially increases and then remains constant. The fluid force rapidly increases just before the particle collides with the bottom wall and then decreases a short time later. Such a rapid change is caused by the lubrication effect. That is, the interstitial pressure drastically increases with decreasing the gap width between a particle and wall and consequently the large repulsive force is exerted on the particle. After the particle collides with the bottom wall, the pullback force, which is caused by the reversible nature of lubrication effect, is exerted and then the force keeps constant. This indicates that the contact force with the wall which balances the gravity and buoyant forces.

Compared Fig.8(a) to (b), it can be seen that overall change in the fluid force agrees well with each other. However, the arrival time to the bottom wall is different between the measured and theoretical results. The measured arrival time is shorter than that of the calculation by Eq.(14). The arrival time can be estimated about 1.2 seconds from the experimental pictures given in Fig. 5 and it supports the validity of the measured results. Therefore it appears that the discrepancy in the arrival time comes from the accumulation of underestimation of the instantaneous fluid force.

Figure 9 indicates the transition of steady and unsteady fluid forces given by Eq.(14) during the early stage of the settling. Just after the particle starts to settle, the accelerated motion is dominant and the unsteady fluid forces (added mass force and history force) are larger than the steady drag. Then the steady drag increases gradually with increasing the particle velocity. Consequently the total fluid force varies according to contributions of these forces. As time sufficiently passes, the force approaches to the sum of the gravity and buoyancy force $\left(m_{p} g-m_{f} g\right) / m_{p} g=1-\rho_{f} / \rho_{p}=0.086\left(m_{f}\right.$ : equivalent mass of fluid). It is found from Fig.9 that our measurement system can resolve such both steady and unsteady fluid forces. At the present stage, we make no further reference to the applicability of each fluid force model. 


\section{Application to Multi-Particle System}

Our measurement system can be applied to multi-particle system. In this section, we demonstrate the application of our system to the settling of particle assemblage in liquid and give some ideas for the measurement of multi-particle system. We made dummy particles which has the same diameter and density as those of the sensor particle. The dummy particle is composed of an outer shell and agar gel. We controlled the density of dummy particles by adding salt in gels.

Figure 10 shows the picture and the weight distribution of the dummy particles. We made 99 dummy particles. In Fig.10 (a), the orange particle is the sensor particle and the white is the dummy particles. It is found from Fig.10 (b) that there is \pm 0.05 gram variation in the mass of dummy particles. We controlled the mass of the sensor particle by adhering of clayey silicone paste on the inner surface of the shell and set it to be 14.55 gram which corresponds to the average mass of dummy particles. The eventual density of sensor particle $\rho_{p}$ is $1056 \mathrm{~kg} / \mathrm{m}^{3}$.

We measured the fluid force acting on aparticle (sensor particle) during the gravitational settling with the dummy particle assemblage in liquid. The test vessel is cylindrical acrylic vessel with 150 $\mathrm{mm}$ in diameter and $1000 \mathrm{~mm}$ in height (See Fig.11). Only the upper part of the test vessel is covered by an outer rectangular vessel. There is a slit on the surface of the test vessel for putting a thin acrylic sheet (release sheet) into it. The thickness of the release sheet is $0.3 \mathrm{~mm}$. The distance between the bottom of the vessel and the release sheet is $755 \mathrm{~mm}$. Silicone oil (density $\rho_{f}=972$ $\mathrm{kg} / \mathrm{m}^{3}$, viscosity $\mu_{f}=63.2 \mathrm{mPa} \cdot \mathrm{s}$ at $14.6^{\circ} \mathrm{C}$ ) is filled into both the test vessel and the outer rectangular vessel. Then the release sheet is put into the slit and the sensor and dummy particles are put above the release sheet. The sensor particle is placed in the center of the bed of dummy particles. After the particle and fluid are at rest, the release sheet is removed rapidly and then the particles start to settle by gravity. The transmitted signal from the sensor particle is received by RX module mounted the outside of the vessel. The settling behavior of particle assemblage is simultaneously recorded by digital video camera. The experiment was performed with changing the total number of particles $N$. 
Figure 11 shows the pictures of the settling behaviors at intervals of 2.0 seconds and the corresponding measured results of the fluid force acting on the sensor particle. As can be seen in the pictures in Fig.11, the particles interact with each other and they show complex trajectories during the settling. It is also found from the pictures that the settling time is longer for larger number of particles. This is caused by the dependence of the hindered settling velocity on particle concentration, i.e., the settling velocity is smaller for denser particle assemblage.

The signal obtained from the sensor particle consists of three kinds of fluctuation. The minute fluctuation is electric noise due to the signal transmission. By comparing to the recorded images, we can recognize that the rapid and steep change of the signal indicates the direct collision of sensor particle with the adjacent particles. The convex (upward) signal is detected when the sensor particle collides with the lower particle, and the concave (downward) signal is detected when the sensor particle collides with the upper particle. The long-time and gradual fluctuation of the signal can be interprleted as the hydrodynamic interaction between particles. We could see it most frequently for $N=60$. This is because the hydrodynamic interaction enlarges at moderate concentration since the particle can move modestly under the influence of the other particles. After the sensor particle collides with the sediment layer, the signal fluctuates largely owing to the collision of the upper particles. The arrival time to the bottom detected by sensor particle agrees well with the recorded images.

From the above results, it is found that the sensor particle can detect both the direct interaction (collision) and the indirect interaction (hydrodynamic interaction) between particles in the solid-liquid system. As mentioned in the previous section, we can measure only the vertical fluid force accurately at the present stage. However, it can be said that the present system achieves a certain result as a prototype of the measurement system of fluid force on a freely-moving particle in liquid. 


\section{CONCLUSIONS}

We have made a "sensor particle" with a built-in wireless sensor for real-time measurement of the fluid force acting on a particle which moves freely in liquid. We applied the external magnetic field which has the same direction as the gravity field and detected the direction of gravitational acceleration by a magnetometer. We measured the force acting on a settling particle toward a solid wall in liquid for checking the accuracy of the measurement system. The obtained instantaneous signal of acceleration (fluid force divided by mass) agreed well with the theoretical prediction of particle motion in liquid. In addition, we applied our system to the gravitational settling of particle assemblage and detected the force acting on a particle in a multi-particle system. 


\section{ACKNOWLEDGMENTS}

This work has been partly supported by the Grant-in-Aid for Young Scientists (B) 15760115, The Ministry of Education, Culture, Sports, Science and Technology, Japan. We would like to thank Mr. Shinichi Itoh (Syoken Sangyo Co., Ltd.) for helpful comments on making the substrate. We also thank Mr. Isao Ohya (Jacom Co., Ltd.) for a lot of variable discussions on this work.

\section{REFERENCES}

Adrian, R.J., 2005. Twenty years of particle image velocimetry. Exp. Fluids 39, 159-169.

Barnocky, G. and Davis, R.H., 1988. Elastrohydrodynamic collision and rebound of spheres: experimental verification. Phys. Fluids 31, 1324-1329.

Brennen, C.E, 2005. Fundamentals of Multiphase Flows. Cambridge University Press, Cambridge.

Chaouki, J., Larachi, F. and Duduković, M.P., 1997. Noninvasive tomographic and velocimetric monitoring of multiphase flow. Ind. Eng. Chem. Res. 36, 4476-4503.

Clift, R., Grace, J.R. and Weber, M.E., 1978. Bubbles, drops, and particles. Dover Pub. Inc., Mineola.

Crowe, C., Sommerfeld, M. and Tsuji, Y., 1998. Multiphase flows with droplets and particles. CRC press, New York.

Gasteuil, Y., Shew, W.L., Gibert, M., Chilla, F., Castaing, B. and Pinton, J.-F., 2007, Lagrangian temperature, velocity, and local heat flux measurement in Rayleigh-Bénard convection. Phys. Rev. Lett. 99, 234302.

Ho, C.-M. and Tai, Y.-C., 1998. Micro-Electro-Mechanical-Systems (MEMS) and fluid flows. Annu. Rev. Fluid Mech. 30, 579-612.

Jackson, J.D., 1999. Classical electrodynamics. 3rd ed., Wiley, New York. 
Joseph, G.G., Zenit, R., Hunt, M.L. and Rosenwinkel, A.M., 2001. Particle-wall collisions in a viscous fluid. J. Fluid Mech. 433, 329-346.

Kawaguchi, T., 2010. MRI measurement of granular flows and fluid-particle flows. Adv. Pow. Tech. $21,235-241$.

Morsi, S.A. and Alexander, A.J., 1972. An investigation of particle trajectories in two-phase flow systems. J. Fluid Mech. 55, 193-208.

Odar, F., 1966. Verification of the proposed equation for calculation of the forces on a sphere accelerating in a viscous fluid. J. Fluid Mech. 25, 591-592.

Odar, F. and Hamilton, W.S., 1964, Forces on a sphere accelerating in a viscous fluid. J. Fluid Mech. 18, 302-314.

Shew, W.L., Gasteuil, Y., Gibert, M., Metz, P. and Pinton, J.-F., 2007, Instrumented tracer for Lagrangian measurements in Rayleigh-Bénard convection. Rev. Sci. Inst. 78, 065105.

Zhang, Q., Huang, C., Jiang, D., Wei, X., Qian, Z. and Wei. F., 2009. Particle measurement sensor for in situ determination of phase structure of fluidized bed. Particuology 7, 175-182.

\section{CAPTIONS}

Fig. 1 Appearance of sensor particle with a built-in sensor system.

Fig. 2 Circuit diagram of sensor and substrate.

Fig. 3 Intensity of wireless signal detection in air and water.

Fig. 4 Output of sensor particle located in magnetic field.

Fig. 5 Measured magnetic field at the central part between the magnets.

Fig. 6 Error of acceleration components caused by inclined magnetic field for $\Delta \theta=8 \mathrm{deg}$.

Fig. 7 Settling behaviors of sensor particle at intervals of 0.2 seconds.

Fig. 8 Fluid force acting on a settling particle. (a) measured fluid force by sensor particle; (b) total 
fluid force calculated by Eq.(5) without gravity and buoyant forces.

Fig. 9 Component of fluid force acting on a settling particle by fluid force model.

Fig. 10 (a) picture of sensor particle (orange) and dummy particles (white); (b) weight distribution of dummy particles.

Fig. 11 Settling behavior and fluid force acting on a particle in assemblage. The pictures show at intervals of 2.0 seconds.

Table 1 Operating conditions and characteristics of sensors and modules 


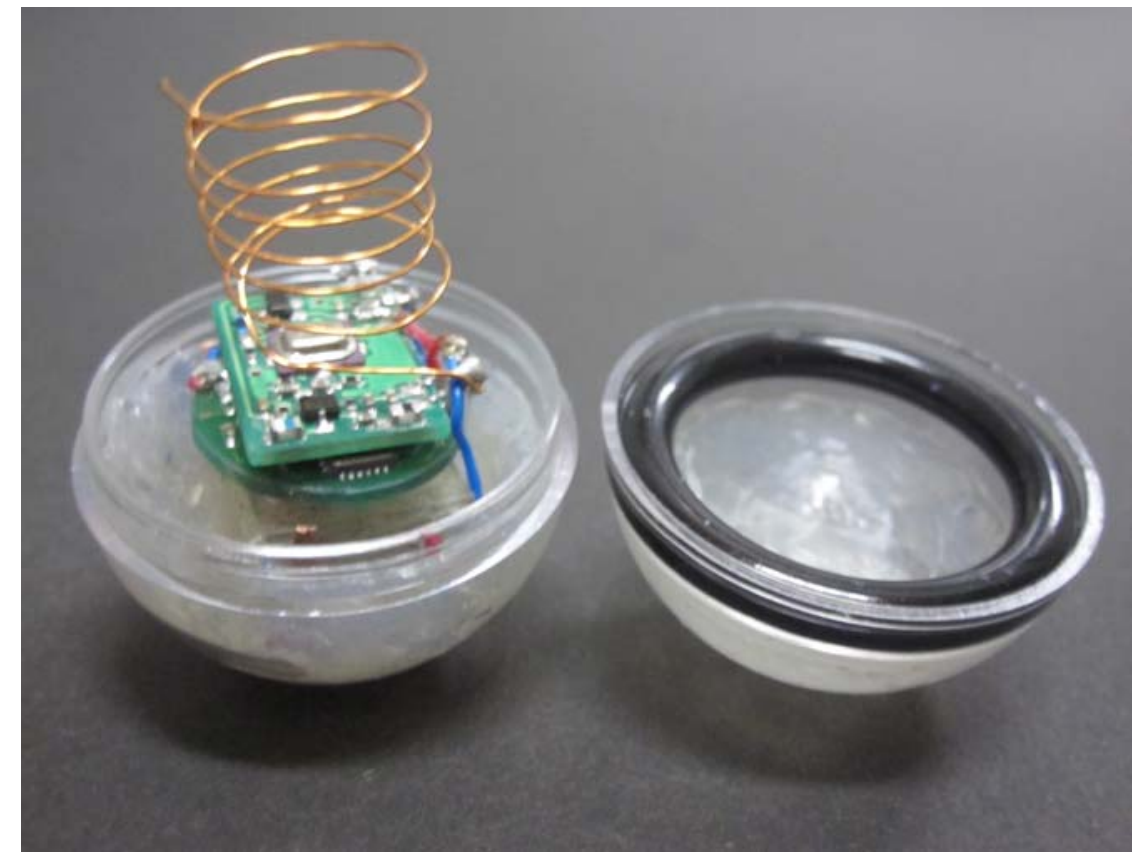

Fig. 1 Appearance of sensor particle with a built-in sensor system. 
Table 1 Operating conditions and characteristics of sensors and modules.

\begin{tabular}{|c|c|c|c|c|c|}
\hline \multicolumn{2}{|c|}{$\begin{array}{c}\text { Acceleration Sensor } \\
\text { (Hitachi Metals, H34C) }\end{array}$} & \multicolumn{2}{|c|}{$\begin{array}{c}\text { Magnetometer } \\
\text { (Aichi-MI, AMI302) }\end{array}$} & \multicolumn{2}{|c|}{$\begin{array}{l}\text { Wireless Module } \\
\text { (ITEC, iTX315S) }\end{array}$} \\
\hline Supply Voltage & 3.0V (Тур.) & SupplyVoltage & 3.0V (Тур.) & SupplyVoltage & 3.0V (Тур.) \\
\hline Size & $3.4 \times 3.7 \times 0.92 \mathrm{~mm}$ & Size & $3.5 \times 4.0 \times 1.45 \mathrm{~mm}$ & Size & $10 \times 10 \times 4.4 \mathrm{~mm}$ \\
\hline Sensitivity & $333 \pm 27 \mathrm{mV} / \mathrm{G}$ & Sensitivity & 3.0mV / $/$ T(Тур.) & Frequency & $315 \mathrm{MHz}$ \\
\hline $\begin{array}{l}\text { Measurement } \\
\text { Range }\end{array}$ & $\pm 3 \mathrm{G}$ & $\begin{array}{c}\text { Dynamic } \\
\text { Range }\end{array}$ & $\pm 0.2 \mathrm{mT}$ & TX Data Rate & $9600 \mathrm{bps}$ \\
\hline $\begin{array}{c}\text { Frequency } \\
\text { Response }\end{array}$ & $\mathrm{DC}-100 \mathrm{~Hz}$ & $\begin{array}{l}\text { Frequency } \\
\text { Response }\end{array}$ & $1 \mathrm{kHz}$ (Max.) & $\begin{array}{l}\text { Modulation } \\
\text { Method }\end{array}$ & FSK \\
\hline
\end{tabular}




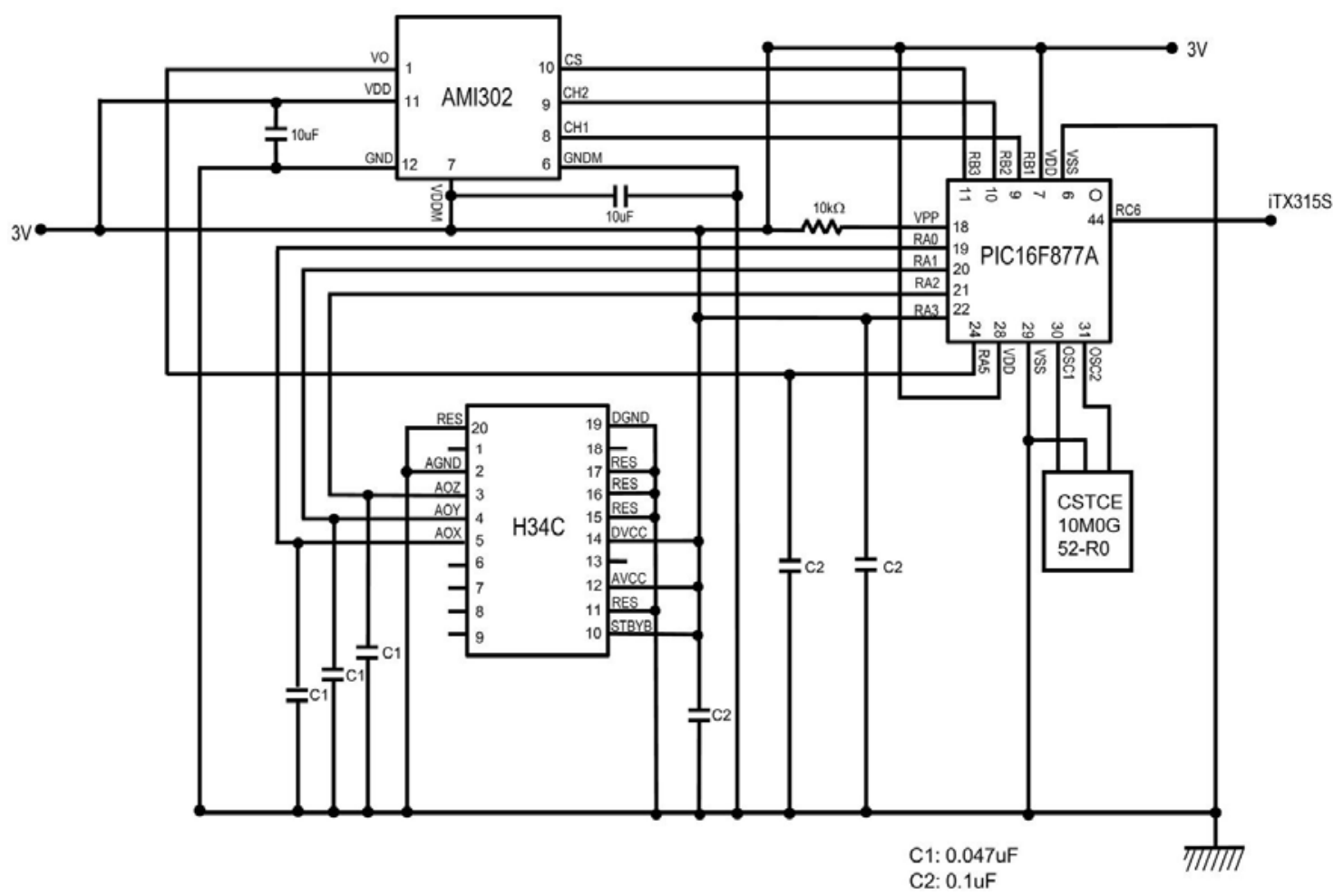

Fig. 2 Circuit diagram of sensor and substrate. 


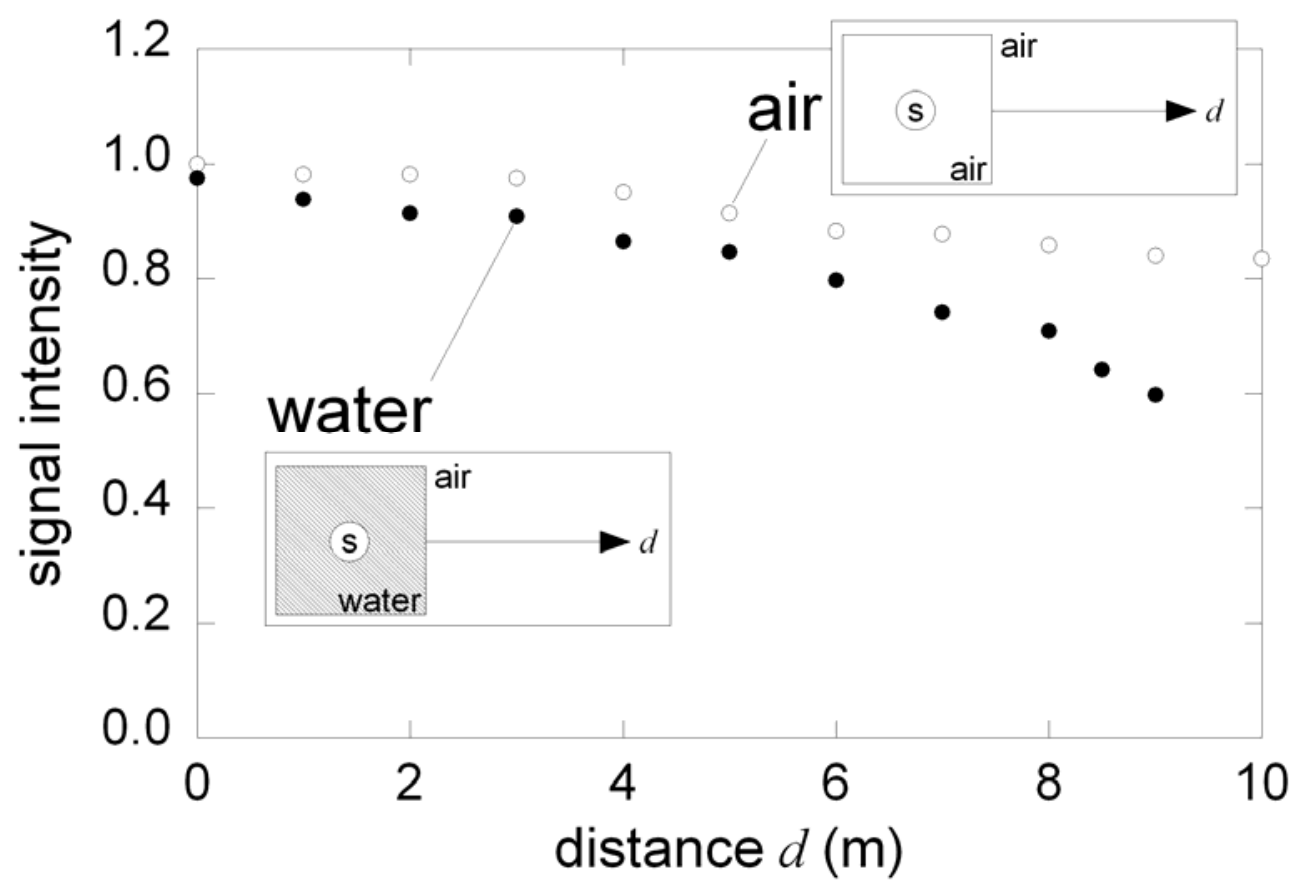

Fig. 3 Intensity of wireless signal detection in air and water. 


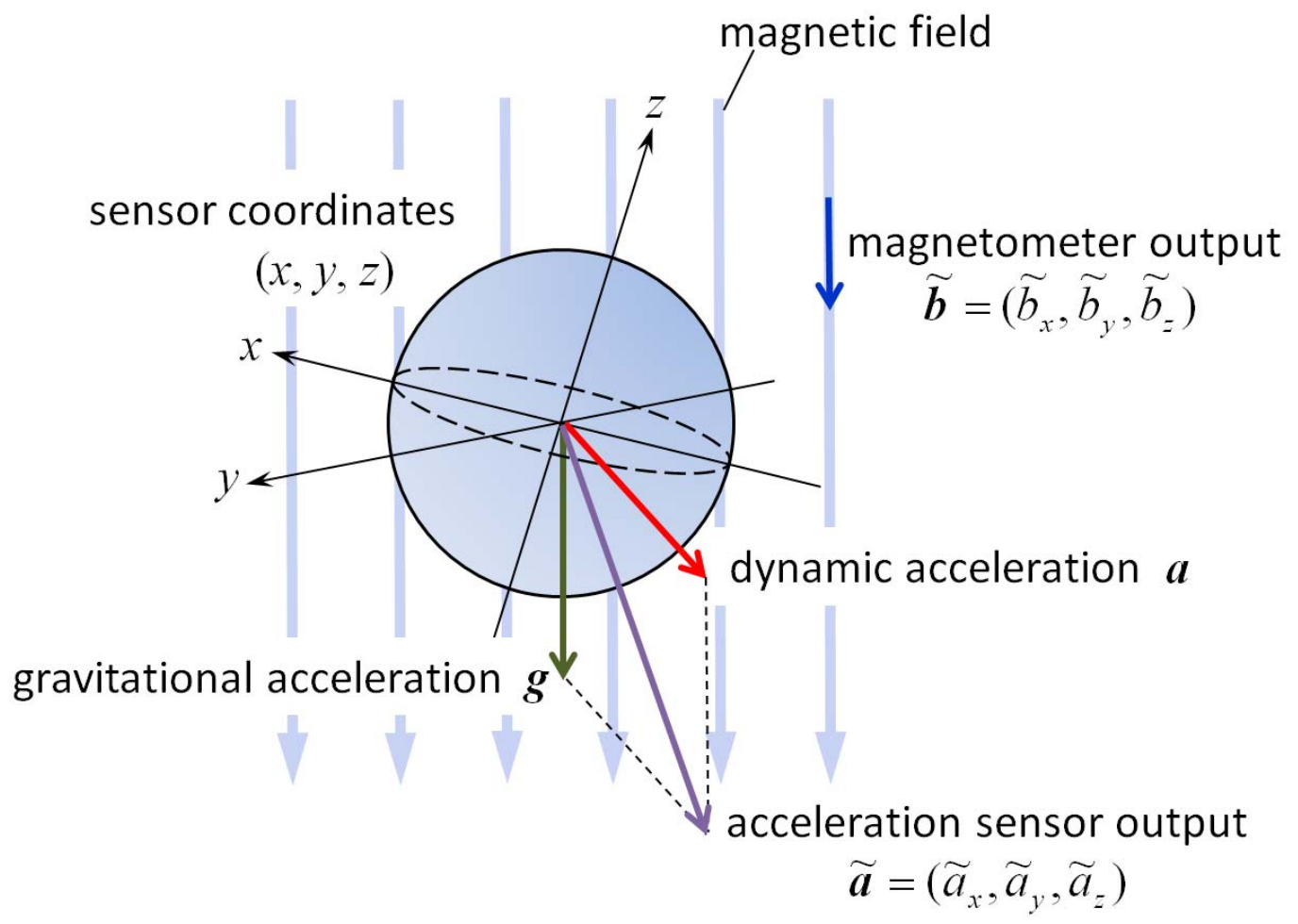

Fig. 4 Output of sensor particle located in magnetic field. 


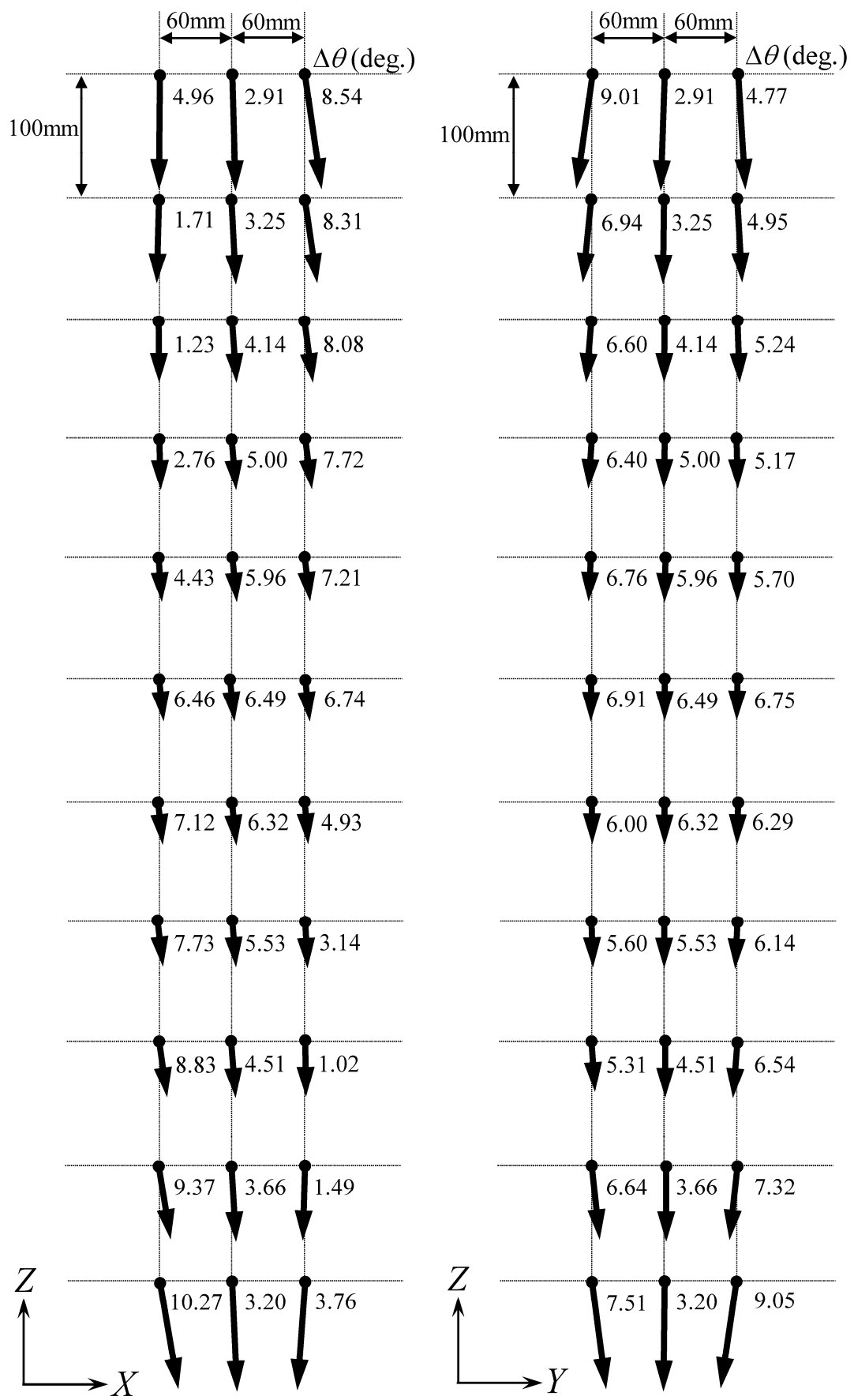

Fig. 5 Measured magnetic field between planar magnets. 

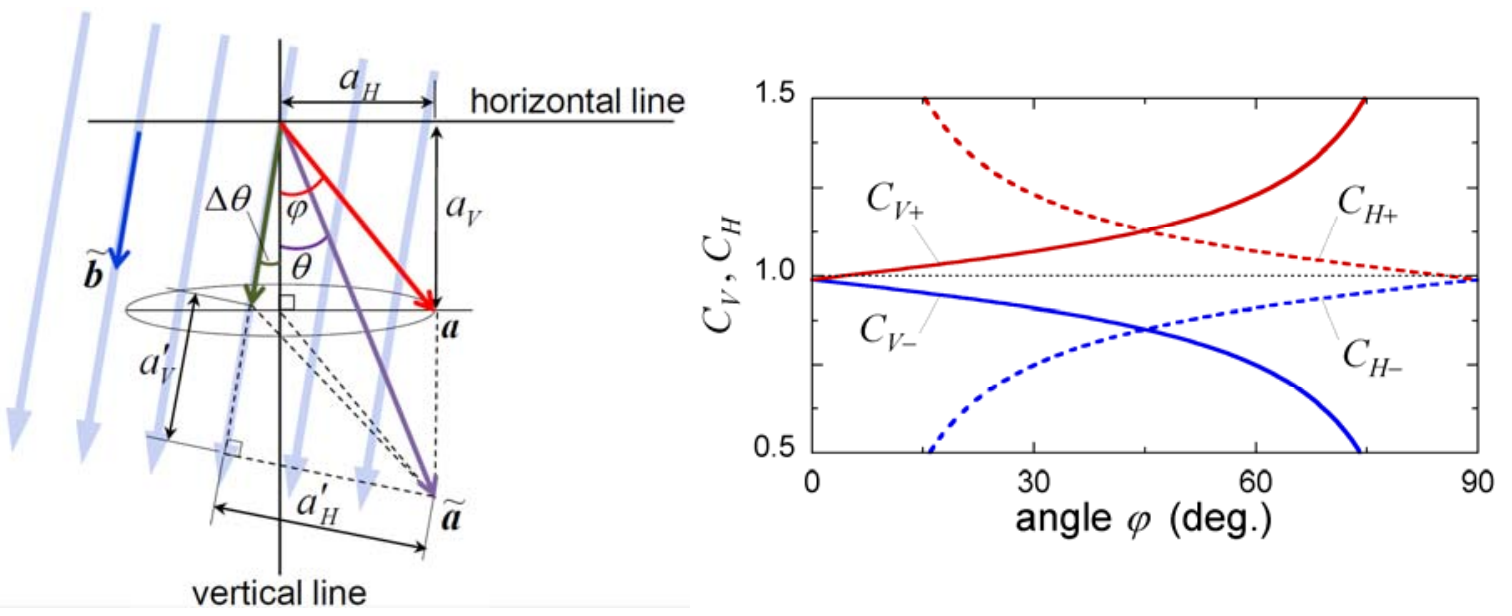

Fig. 6 Error of acceleration components caused by inclined magnetic field for $\Delta \theta=8 \mathrm{deg}$. 


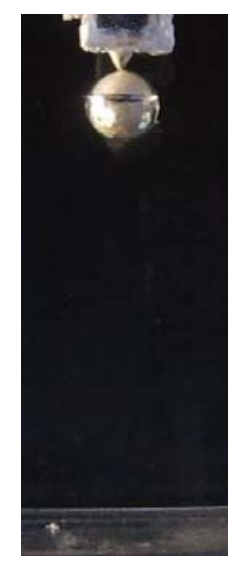

$t=0$

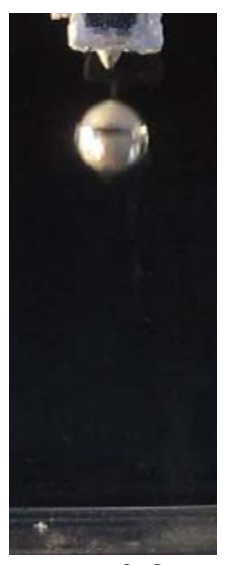

$t=0.2$

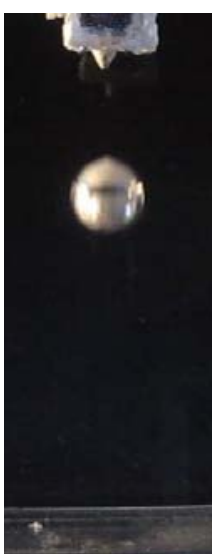

$t=0.4$

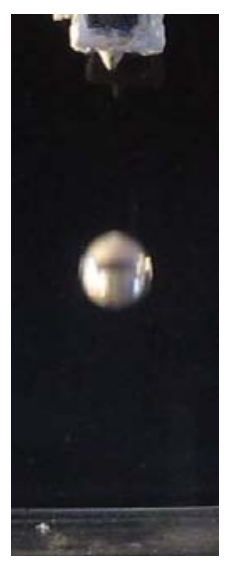

$t=0.6$

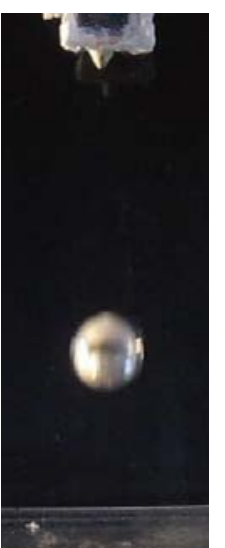

$t=0.8$

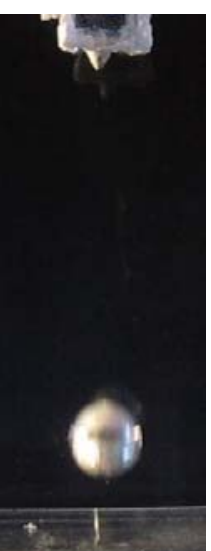

$t=1.0$

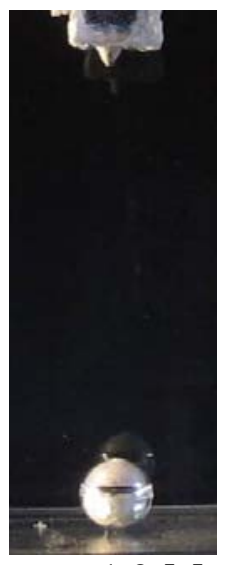

$t=1.2[\mathrm{~s}]$

Fig. 7 Settling behaviors of sensor particle at intervals of 0.2 seconds. 


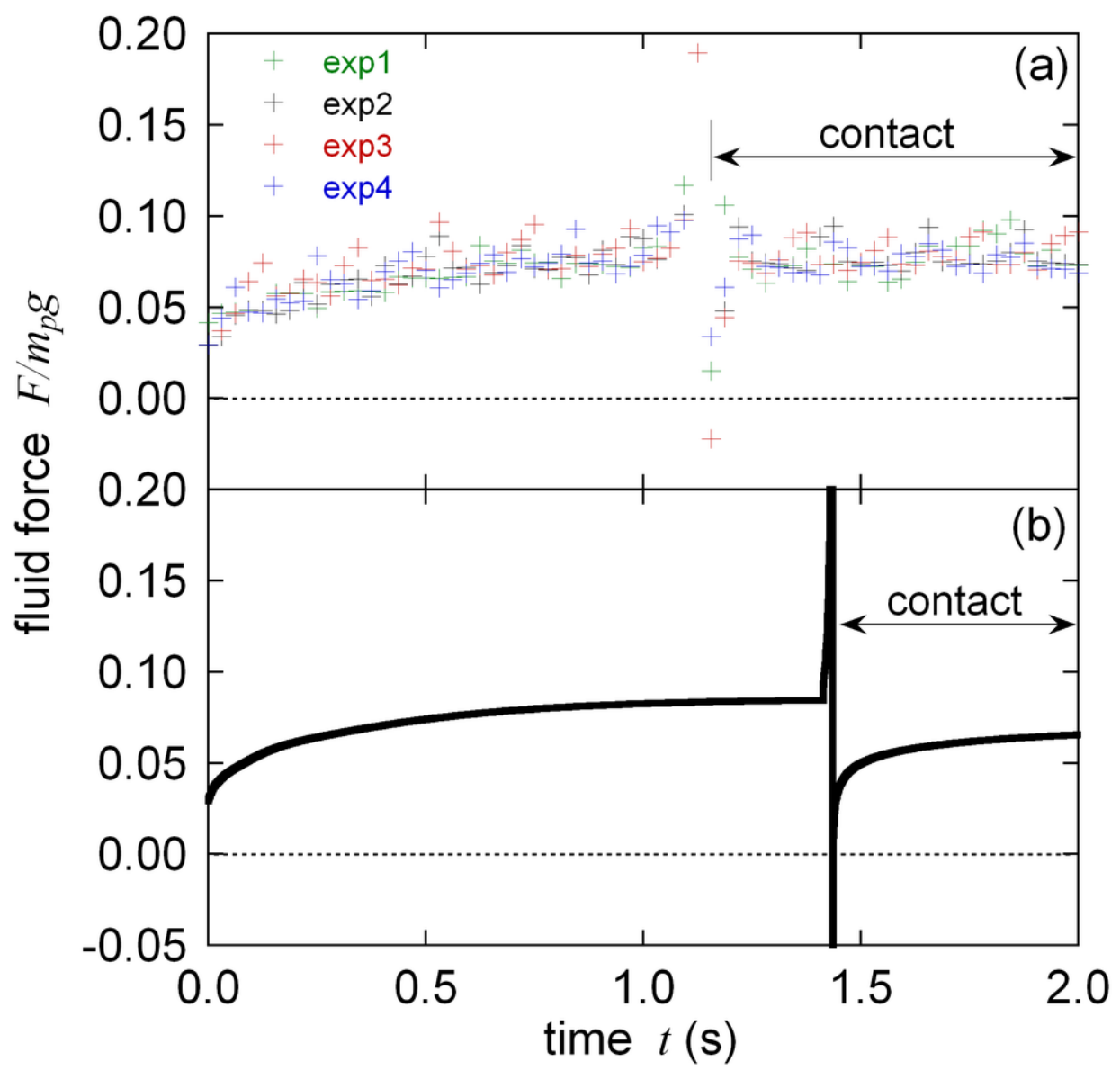

Fig. 8 Fluid force acting on a settling particle. (a) measured fluid force by sensor particle; (b) total fluid force calculated by Eq.(5) without gravity and buoyant forces. 


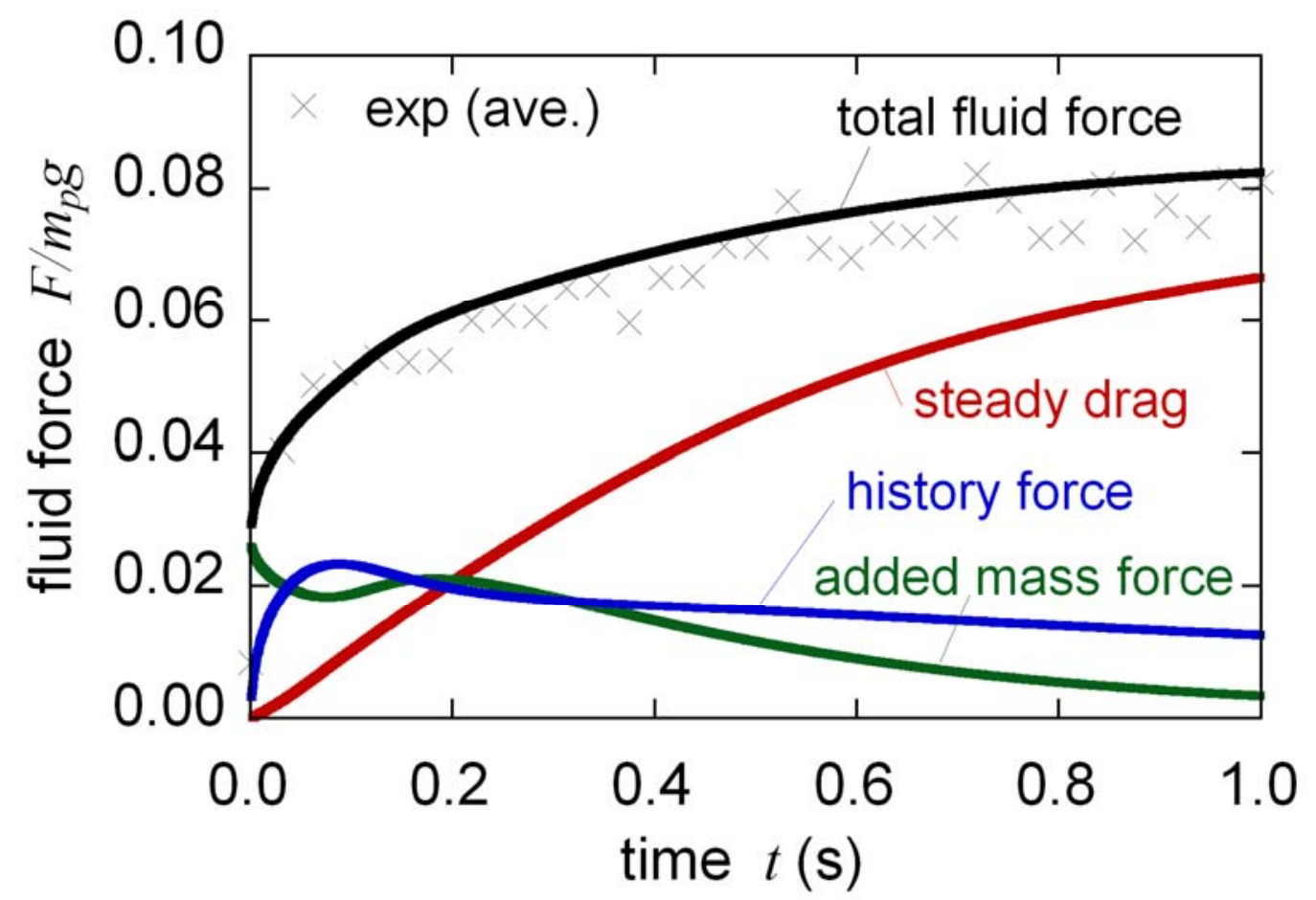

Fig. 9 Component of fluid force acting on a settling particle by fluid force model. 

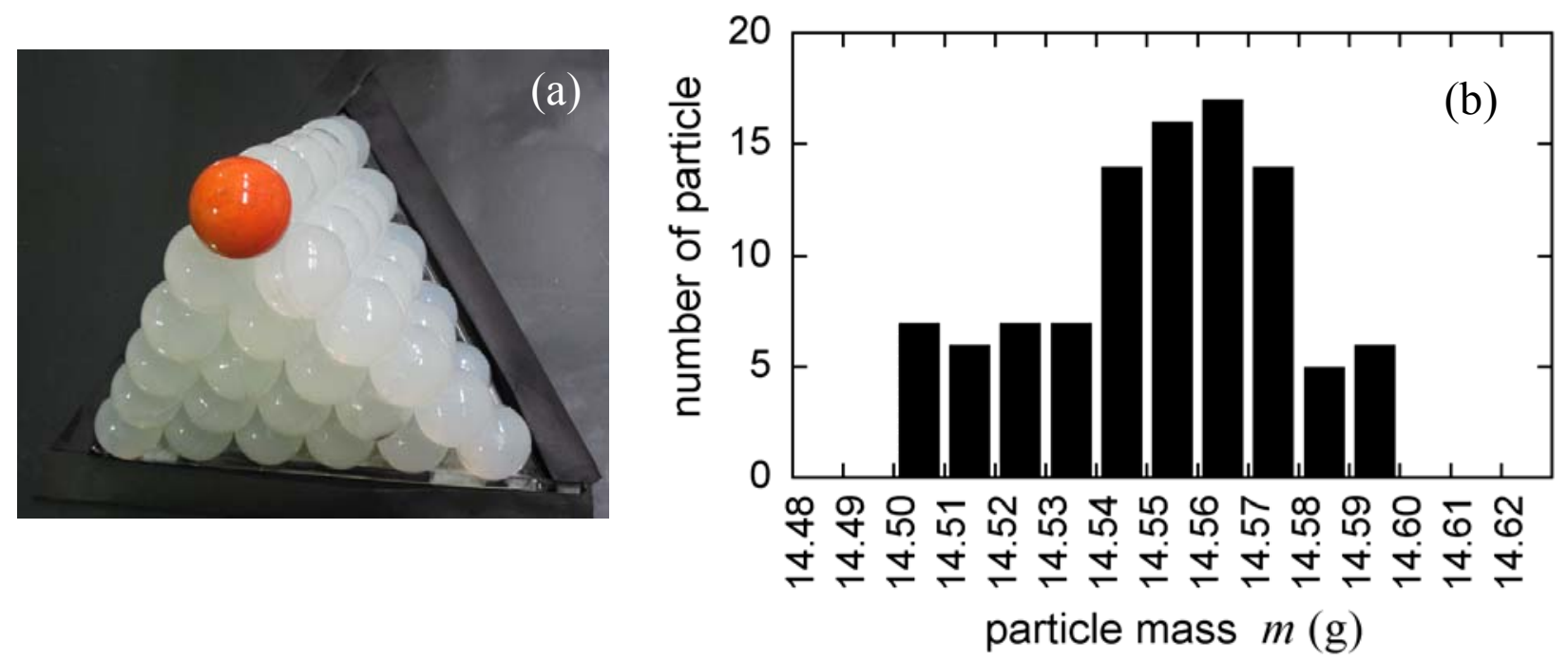

Fig. 10 (a) picture of sensor particle (orange) and dummy particles (white); (b) weight distribution of dummy particles. 
(a) $N=40$
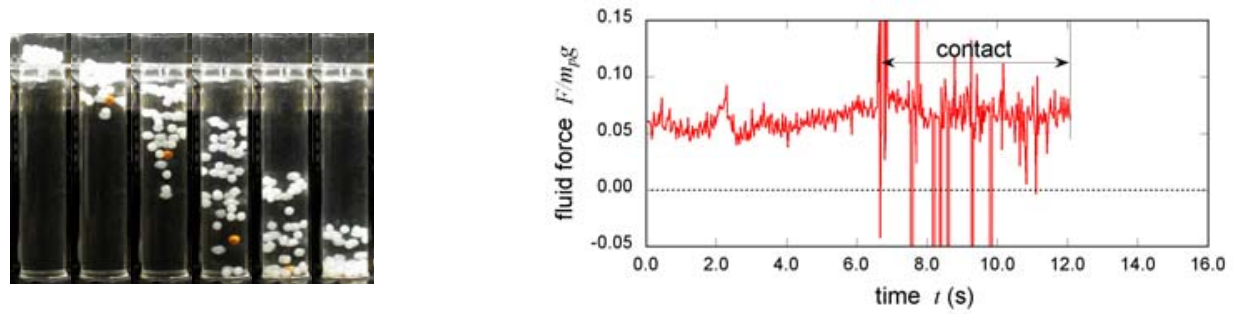

(b) $N=60$
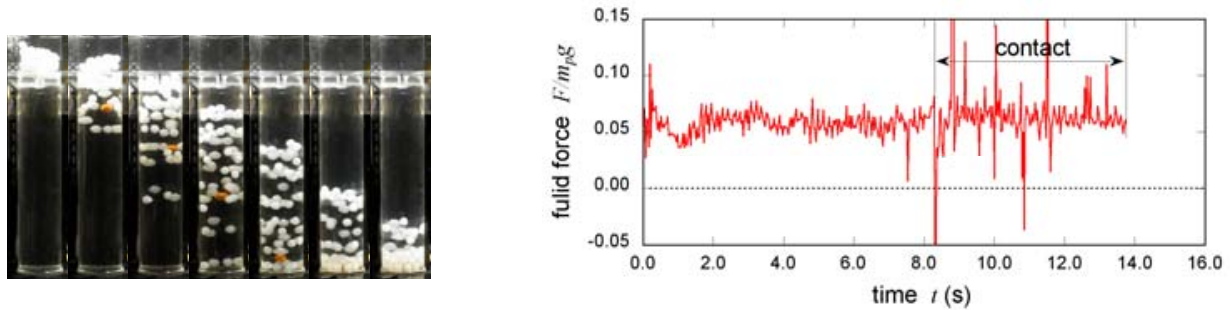

(c) $N=100$
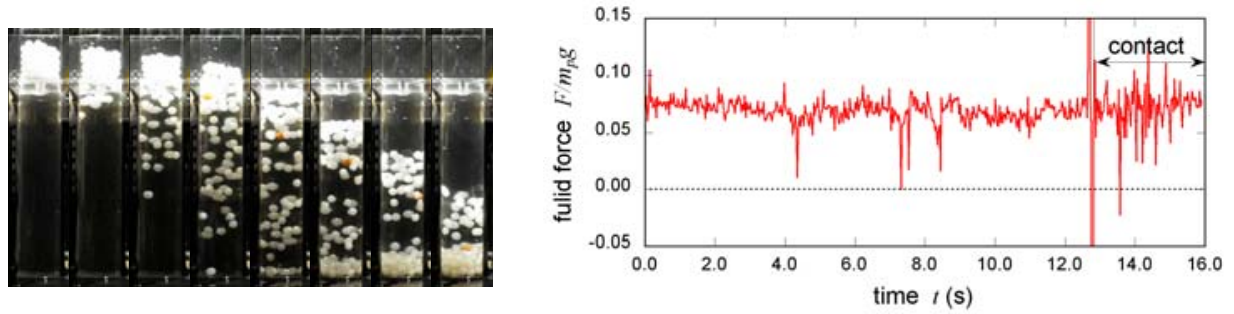

Fig. 11 Settling behavior and fluid force acting on a particle in assemblage. The pictures show at intervals of 2.0 seconds. 